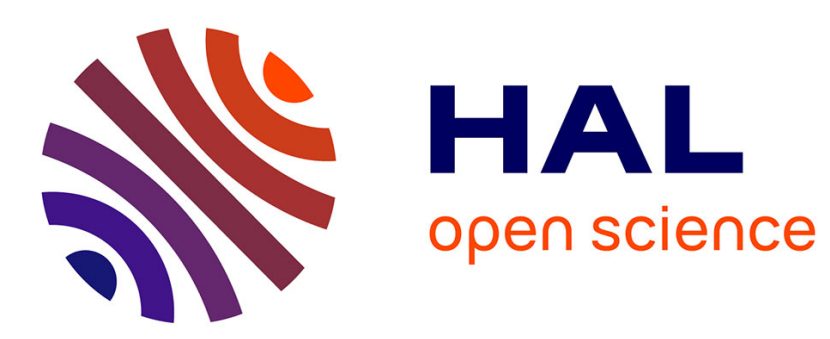

\title{
Linearly forced isotropic turbulence at low Reynolds numbers
}

\author{
Wouter J.T. Bos, Faouzi Laadhari, Wesley Agoua
}

\section{To cite this version:}

Wouter J.T. Bos, Faouzi Laadhari, Wesley Agoua. Linearly forced isotropic turbulence at low Reynolds numbers. Physical Review E , 2020, 102, 10.1103/PhysRevE.102.033105 . hal-02933727

\section{HAL Id: hal-02933727 \\ https://hal.science/hal-02933727}

Submitted on 11 Sep 2020

HAL is a multi-disciplinary open access archive for the deposit and dissemination of scientific research documents, whether they are published or not. The documents may come from teaching and research institutions in France or abroad, or from public or private research centers.
L'archive ouverte pluridisciplinaire HAL, est destinée au dépôt et à la diffusion de documents scientifiques de niveau recherche, publiés ou non, émanant des établissements d'enseignement et de recherche français ou étrangers, des laboratoires publics ou privés. 


\title{
Linearly forced isotropic turbulence at low Reynolds numbers
}

\author{
Wouter J. T. Bos $\odot,{ }^{*}$ Faouzi Laadhari $\odot$, and Wesley Agoua \\ LMFA-Ecole Centrale de Lyon, CNRS-Univ. Claude Bernard Lyon 1, Univ. Lyon, 36 Avenue Guy de Collongue, F-69134 Ecully, France
}

(Received 28 November 2019; accepted 19 August 2020; published 8 September 2020)

\begin{abstract}
We investigate the forcing strength needed to sustain a flow using linear forcing. A critical Reynolds number $R_{c}$ is determined, based on the longest wavelength allowed by the system, the forcing strength and the viscosity. A simple model is proposed for the dissipation rate, leading to a closed expression for the kinetic energy of the flow as a function of the Reynolds number. The dissipation model and the prediction for the kinetic energy are assessed using direct numerical simulations and two-point closure integrations. An analysis of the dissipationrate equation and the triadic structure of the nonlinear transfer allows to refine the model in order to reproduce the low-Reynolds-number asymptotic behavior, where the kinetic energy is proportional to $R-R_{c}$.
\end{abstract}

DOI: 10.1103/PhysRevE.102.033105

\section{TRANSITION AND INSTABILITY IN ISOTROPIC TURBULENCE}

Transition in shear flows and the modeling of isotropic turbulence are two seemingly different research subjects. Recently, however, it was realized that isotropic turbulence and the laminar-turbulent transition in wall-bounded shear flows share certain features [1]. For instance, it was shown that relaminarization, a feature generally associated with shear flows [2] such as flow through pipes driven by a pressure gradient, can also be observed in statistically isotropic periodic box turbulence. In Ref. [1] the forcing that was used to excite the Navier-Stokes equations allowed the existence of large-scale, linearly stable, and spatially simple solutions. When turbulence was triggered through the initial conditions (or a finite amplitude perturbation), the distribution of turbulent lifetimes before the flow "relaminarizes" was observed to be exponential and the characteristic timescale increased superexponentially with the Reynolds number, as is also the case in pipe flow. These observations established a phenomenological connection between isotropic turbulence and certain wall-bounded shear flows.

In the present investigation we further explore the transitional dynamics of isotropic turbulence. The dynamics of the system we consider, using a distinct forcing, is different in nature from the transition observed in Ref. [1]. We thereby illustrate that in isotropic turbulence the transition from a laminar to a turbulent state is not universal and can depend on the type of forcing. In particular, we will show that in the case of linear forcing [3] a critical Reynolds number exists above which turbulence can be sustained. The quantity that we will evaluate is the kinetic energy of the flow and the present approach is thereby related to the classical energy stability approach [4]. The originality in the present work is that we do not only determine the critical Reynolds number below which energy always decays, but that we also model the dependence of the kinetic energy on the Reynolds number, for all Reynolds numbers from the onset up to the fully developed turbulent regime.

In periodic or closed systems, the global kinetic energy is fully determined by the production and dissipation mechanisms. In linearly forced flow the production mechanism is a closed (linear) function of the kinetic energy itself, but the dissipation term is unclosed and, if one wants to predict the behavior of the kinetic energy, this term needs to be modeled. We use an existing model for the dissipation and obtain thereby a closed expression for the kinetic energy as a function of the Reynolds number. The predictions are tested using direct numerical simulations (DNS) and numerical integration of closure equations. It is observed that the model for the kinetic energy gives only a rough description of the data. Subsequently we show how analytical considerations allow to refine the model in order to get a correct description of the energy balance near the critical Reynolds number.

In the following section we discuss linearly forced turbulence and give a first estimate of the kinetic energy as a function of the Reynolds number involving two model constants. Then in Sec. III we consider the Fourier-spectra of the kinetic energy and show how we can determine these model constants. We then present numerical results in Sec. IV. Unexpected observations in these results are explained in Sec. V where a refined model is proposed to describe the data accurately. In Sec. VI we discuss the results and suggest that the understanding of the kinetic energy balance near the onset might prove important in order to better understand the physics of transition in certain shear flows.

\section{LINEARLY FORCED FLOW}

The system we consider is

\footnotetext{
*wouter.bos@ec-lyon.fr
} 
where $\boldsymbol{u}$ is the velocity, $\mathcal{P}$ pressure (divided by density), $v$ the kinematic viscosity, the last term being the linear forcing term introduced in Ref. [3] and further investigated in the context of high-Reynolds-number turbulence in Refs. [5,6]. The physical interest of this term can be seen if one considers the evolution of velocity fluctuations in the presence of a mean shear. In that case, the equation for the fluctuations $\boldsymbol{u}^{\prime}$, contains a force term $\boldsymbol{u}^{\prime} \cdot \nabla \overline{\boldsymbol{u}}$, where $\nabla \overline{\boldsymbol{u}}$ is the mean velocity gradient. The linear forcing can be seen as a way to investigate such a natural manner of forcing in the context of isotropic turbulence, avoiding thus the complexity induced by anisotropy and inhomogeneity. The parameter $\alpha$ in the forcing can then be associated with the strength of the mean-velocity gradient $\nabla \overline{\boldsymbol{u}}$ in more realistic shear flows, even though in such flows the forcing term has in general a tensorial structure.

It is the simplicity of the present approach, compared to forcing by shear, which is important in the present study, since it allows one to obtain a deeper understanding of the influence of purely linear mechanisms on the transition to turbulence in the geometrically simplest setting of isotropic turbulence. Even though this forcing is clearly not representative of all possible physical forcing mechanisms, the profound understanding of the role of linear forcing on isotropic turbulence can then inspire investigations of more complex flows.

For instance, in channel flow an ongoing question is the precise role of linear mechanisms in the generation and sustainment of the turbulent fluctuations and the formation of coherent structures [7-9]. Another issue is the transition to turbulence in such flows on which we will come back later. Further motivations, beyond its resemblance with natural forcing by shear and the simplicity of the approach, can be found in the original work by Lundgren [3].

We write the kinetic energy balance for (1),

$$
\frac{d K}{d t}=P-\epsilon,
$$

where the kinetic energy $K$, production $P$ and dissipation $\epsilon$ are defined by

$$
\begin{gathered}
K=\left\langle\|\boldsymbol{u}\|^{2}\right\rangle / 2, \\
\epsilon=-v\langle\boldsymbol{u} \cdot \Delta \boldsymbol{u}\rangle, \\
P=2 \alpha K,
\end{gathered}
$$

and where the brackets denote an ensemble average. A reasonable estimate of the dissipation, both for low and for high Reynolds numbers [10], is

$$
\epsilon=\frac{K^{3 / 2}}{L}\left(\frac{C_{v}}{R_{K}}+C_{\epsilon}\right), \quad(\text { Model } 1)
$$

where

$$
R_{K}=\frac{L K^{1 / 2}}{v},
$$

and $L$ is a typical length scale, characterizing the large flow scales. In the following, expression (6) will be denoted by Model 1. The model contains two terms. The first, linear term, contains the constant $C_{\nu}$, and is dominant for low values of the kinetic energy. The constant $C_{v}$ will be shown to set the critical Reynolds number below which kinetic energy will always decay. At large values of $R_{K}$, the influence of this term disappears and the other term dominates. The constant $C_{\epsilon}$ in this nonlinear contribution sets the asymptotic value of the normalized dissipation rate $\epsilon L / K^{3 / 2}$ [11] and represents thereby the dissipative anomaly [12], i.e., a nonvanishing dissipation when the viscosity tends to zero.

It is indeed observed that $\epsilon L / K^{3 / 2}$ tends to a constant at high Reynolds numbers for a given flow [13], but its value can depend on the large-scale structure of the energetic scales [14], the presence of helicity [15], or the instationarity (as observed in Ref. [16] and explained in Ref. [17]). Model 1 was rigorously derived as an upperbound for turbulent dissipation in the Navier-Stokes equations both for static forcing [18,19] and dynamic forcing [15]. The low Reynolds limit of Model 1 was shown to quite accurately describe the results of simulations and closure in references [10,14]. Furthermore, the same expression was proposed in Ref. [20] in order to better understand the dynamics of Taylor-Couette flows and accretion disks. A refinement of this expression, adding terms proportional to $R_{K}^{-2}$ was proposed in Ref. [21], in the context of magnetohydrodynamic turbulence and we will come back to possible refinements in section $\mathrm{V}$.

Using Model 1 for the dissipation in the energy balance (2) we find

$$
\frac{d K}{d t}=\left(2 \alpha-C_{v} \frac{v}{L^{2}}\right) K-C_{\epsilon} \frac{K^{3 / 2}}{L} .
$$

We can readily determine the steady state solutions of this equation. One solution is $K=0$ and the other solution is

$$
\frac{K}{(\alpha L)^{2}}=\frac{4}{C_{\epsilon}^{2}}\left(1-\frac{R_{c}}{R}\right)^{2}
$$

where

$$
R=\frac{\alpha L^{2}}{v} \text { and } R_{c}=C_{v} / 2 .
$$

The independent control parameter of our system is therefore the Reynolds number $R$. Beyond a critical value, $R>R_{c}$, only solution (9) is linearly stable and the kinetic energy will become nonzero in the presence of infinitesimal perturbations. We observe thus a bifurcation in this isotropic system on the level of the volume-averaged or ensemble-averaged quantities. The solution depends on the parameters $L, \alpha, v$ and contains the constants $C_{v}$ and $C_{\epsilon}$. The influence of the three parameters on the turbulence is combined in the forcingbased Reynolds number, which is the control parameter of our system. The physical interpretation of the two model constants is discussed in the following section. Indeed, these constants are related to a critical Reynolds number determined by the domain size and the Kolmogorov constant, respectively.

\section{SPECTRAL ANALYSIS OF THE SYSTEM}

We consider a cubic periodic domain, of size $L_{D}=2 \pi$, so that the smallest wave number is $\kappa_{0}=1$. The energy spectrum is defined as the three-dimensional shell-averaged kinetic energy density so that its integral is $\int_{0}^{\infty} E(\kappa) d \kappa=K$ and its dynamics is given by Lin's equation (see, for instance, Ref. [22] for a derivation),

$$
\partial_{t} E(\kappa)=F(\kappa)+T(\kappa)-2 v \kappa^{2} E(\kappa) .
$$


The three terms on the right-hand side represent production, spectral transfer and dissipation, respectively. The production is

$$
F(\kappa)=2 \alpha E(\kappa) .
$$

The integral of (11) yields (2), since

$$
\int_{0}^{\infty} T(\kappa) d \kappa=0
$$

by energy conservation and

$$
\int_{0}^{\infty} 2 v \kappa^{2} E(\kappa) d \kappa=\epsilon
$$

\section{A. Low Reynolds number}

Let us first discuss the viscous limit. When the Reynolds number is small enough, the nonlinear transfer is smaller than the viscous term for all modes,

$$
|T(\kappa)| \ll 2 v \kappa^{2} E(\kappa) .
$$

Clearly, the most unstable mode is the mode with the smallest wave number $\kappa_{0}$, so that, ignoring the transfer and focusing on the most unstable mode,

$$
\partial_{t} E\left(\kappa_{0}\right) \approx 2\left(\alpha-v \kappa_{0}^{2}\right) E\left(\kappa_{0}\right) .
$$

We have thus energy growth for

$$
\frac{\alpha}{v \kappa_{0}^{2}}>1,
$$

or, introducing an arbitrary length scale $L$,

$$
\frac{\alpha L^{2}}{v}>\left(L \kappa_{0}\right)^{2} \equiv R_{c} \text {. }
$$

In the following we will choose the characteristic length scale as $L=\kappa_{0}^{-1}$, which sets the critical Reynolds number $R_{c}=1$ and therefore the value of $C_{v}$ in expression Model 1 [expression (6)] and in Eq. (10) is $C_{v}=2$. [Note that if instead we use the domain size $L=L_{D}$, the value of the critical Reynolds number would change to $R_{c}=(2 \pi)^{2}$ and the value of $C_{v} \equiv$ $2 R_{c}$ would change accordingly.]

The spectral analysis has therefore allowed to determine one of the two model constants. We have thereby obtained a dissipation model which depends only on the constant $C_{\epsilon}$, which is determined by the high-Reynolds-number dynamics, which we will consider now.

\section{B. High Reynolds number}

At very high Reynolds numbers, a Kolmogorov spectrum will develop,

$$
E(\kappa)=C_{K} \epsilon^{2 / 3} \kappa^{-5 / 3} g(\kappa \zeta),
$$

with $\zeta=v^{3 / 4} \epsilon^{-1 / 4}$ the Kolmogorov length scale, $C_{K}$ the Kolmogorov constant, and $g(x)$ a function which tends to $g(x)=$ 1 for $x \ll 1$ and which drops to zero rapidly for $x>1$. Since in a steady state the production equals the dissipation, and the production is given by $2 \alpha K$, we find for large Reynolds numbers by integrating (19) from $\kappa=\kappa_{0}$ to $\kappa=\zeta^{-1}$,

$$
K \approx \frac{3}{2} C_{K}(2 \alpha K)^{2 / 3} \kappa_{0}^{-2 / 3} .
$$

so that the steady-state kinetic energy is given by

$$
K=\frac{27}{2} C_{K}^{3}\left(\frac{\alpha}{\kappa_{0}}\right)^{2} .
$$

The normalized dissipation is

$$
\frac{\epsilon L}{K^{3 / 2}}=\left(\frac{3}{2} C_{K}\right)^{-3 / 2} L \kappa_{0} .
$$

This relation states that for the choice $L=\kappa_{0}^{-1}$, the value of $C_{\epsilon}=\left(\frac{3}{2} C_{K}\right)^{-3 / 2}$. This expression assumes that the Kolmogorov spectrum starts directly at $\kappa_{0}$. We will see that this is not exactly the case, so that this relation between $C_{\epsilon}$ and $C_{K}$, is only approximately valid. However, it is expected that the normalized dissipation tends to a constant and that its order of magnitude is given by (22).

\section{NUMERICAL RESULTS}

In order to assess expression (9), we carry out both direct numerical simulations of periodic box turbulence and numerical integration of the eddy-damped quasinormal Markovian (EDQNM) closure equations. We will first discuss the numerical method and parameters and after that we will present the results.

\section{A. Numerical methods and setup}

We perform a series of 17 direct numerical simulations of Eqs. (1) in a cubic three-dimensional periodic domain of size $2 \pi$, using a standard pseudospectral solver. Dealiasing is performed by applying the $2 / 3$-rule and the time integration is performed using a third-order Adams-Bashforth scheme with exact integration of the viscous and forcing terms. The initial velocity field consists of incompressible random noise with an initial value of the kinetic energy of $K=20$. The parameters of our simulations and steady-state values of certain quantities are summarized in Table I.

In addition to these simulations we have numerically integrated the EDQNM model [23]. This closure was recently used to study linearly forced isotropic turbulence [24] and was shown to yield correct behavior for this kind of flow at high Reynolds numbers. We solve equation (11) with

$$
\begin{aligned}
T(\kappa)= & \iint_{\Delta}\left(x y+z^{3}\right) \Theta(\kappa, p, q) \frac{E(q)}{q} \\
& \times\left[\kappa^{2} E(p)-p^{2} E(\kappa)\right] d p d q .
\end{aligned}
$$

The integration domain $\Delta$ consists of the subspace in the $p-q$ plane where line segments of length $\kappa, p, q$ can form a triangle. The cosines of the angles inside this triangle, opposite to the sides $\kappa, p, q$ are denoted $x, y, z$, respectively. The characteristic triad timescale is

$$
\Theta(\kappa, p, q)=\left[v\left(\kappa^{2}+p^{2}+q^{2}\right)+\eta(\kappa)+\eta(p)+\eta(q)\right]^{-1}
$$

with $\eta(\kappa)=\lambda_{D}\left[\int_{0}^{\kappa} s^{2} E(s) d s\right]^{1 / 2}$ and $\lambda_{D}$ a constant, determining the strength of the eddy damping, and thereby setting the Kolmogorov constant. The formulation of the model is standard and we refer to Ref. [25] for details. We have not 
TABLE I. Parameters of the DNSs. The forcing strength is fixed at $\alpha=1$, domain size $L_{D}=2 \pi$, reference length scale $L=$ $L_{D} / 2 \pi=1$. Resolution is $128^{3}$. The numerical value of the Reynolds number is here therefore $R=v^{-1}$. The dissipation in the steady state is equal to $\epsilon=2 \alpha K$. The value $K=0$ means zero to machine precision, and $K=\infty$ means unbounded growth until numerical instabilities appear.

\begin{tabular}{lccc}
\hline \hline$R$ & $K$ & $R_{\lambda}$ & $R_{K}$ \\
\hline 0.95 & 0 & - & - \\
1.3 & $\infty$ & - & - \\
2 & 9.18 & 7.8 & 5.6 \\
2.5 & 12.8 & 10.3 & 8.0 \\
3 & 16.2 & 12.7 & 10.7 \\
4 & 17.35 & 15.2 & 15.6 \\
5 & 20.07 & 18.3 & 21.4 \\
6 & 18.92 & 19.5 & 26.5 \\
8 & 19.13 & 22.6 & 38.0 \\
10 & 18.81 & 25.0 & 50.0 \\
15 & 18.43 & 30.4 & 82.6 \\
20 & 16.66 & 33.3 & 115 \\
30 & 16.01 & 40.0 & 190 \\
40 & 16.12 & 46.4 & 272 \\
50 & 15.77 & 51.3 & 358 \\
60 & 17 & 58.3 & 458 \\
70 & 16.61 & 62.3 & 552 \\
\hline \hline
\end{tabular}

added the influence of the forcing term to the timescale $\Theta$, since this can violate the stability of the closure. An investigation showing how to implement the effect of forcing on the transfer in Markovian closures would be valuable, but is outside the scope of the present investigation. The eddy-damping parameter is set to $\lambda_{D}=0.42$ in order to fit the results for the kinetic energy at high Reynolds numbers to the DNS. Simulations were carried out with a logarithmic discretization with 47 modes per decade on a grid ranging from $\kappa=\kappa_{0}=1$ to $\kappa \zeta=5$, with $\zeta$ the Kolmogorov length scale (defined in the previous section). In the following only steady-state results are reported, which are independent of the initial conditions. The parameters $\alpha$ and $L$ are set to unity and the Reynolds number is varied by changing the viscosity from $v=1$ to 0.01 , corresponding to Reynolds numbers $R=\alpha L^{2} / v$ in the range $[1,100]$. For comparison with literature, we give the relation between $R$ and the Taylor-scale Reynolds number,

$$
R_{\lambda}=\sqrt{\frac{10}{3}} \frac{K^{1 / 2}}{\sqrt{\alpha \nu}}=\sqrt{\frac{10}{3}} R_{K} R^{-1 / 2},
$$

which is derived assuming a statistically steady state.

\section{B. Results}

In Fig. 1(a) we show the DNS results for the time evolution of the kinetic energy for different values of the Reynolds number. In particular, we observe for $R=0.95$ a decrease of the kinetic energy. This decrease is observed for all values of the Reynolds number $R<1$. For $R=1.3$ we observe an unbounded increase of the kinetic energy, until numerically the solution cannot be resolved anymore. This behavior was not anticipated in the foregoing. For values $R>2$, the kinetic energy attains a statistically steady state. For long times,

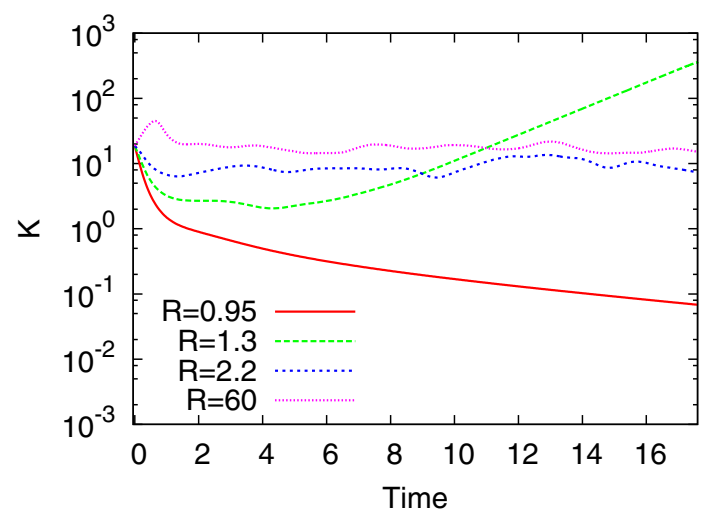

(a)

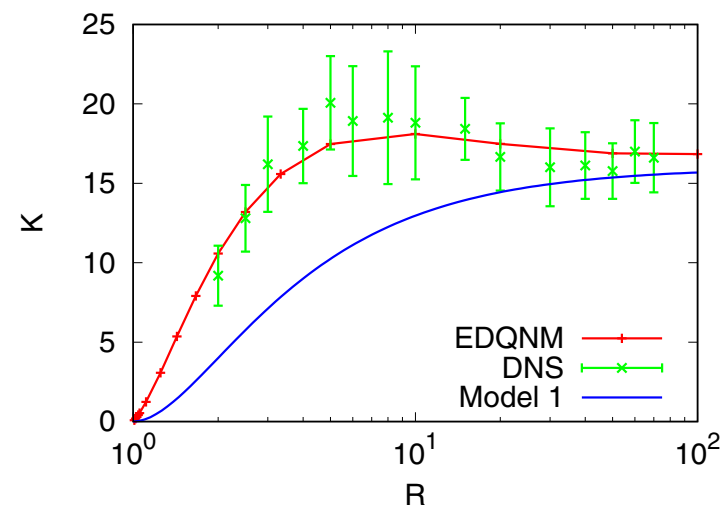

(b)

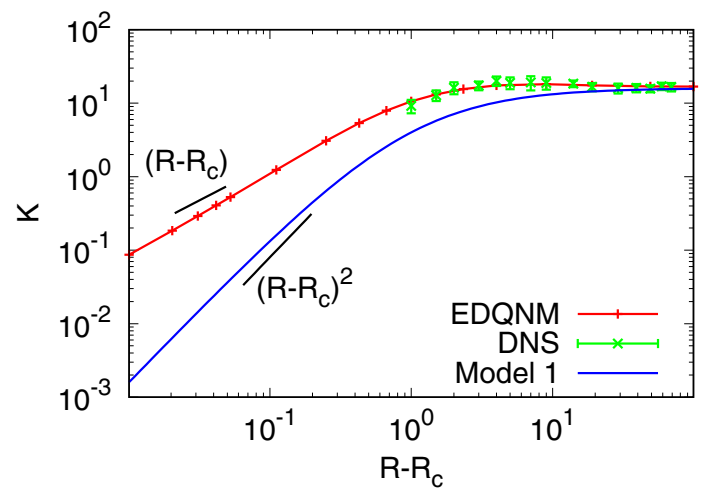

(c)

FIG. 1. (a) Time evolution of the box-averaged kinetic energy for several distinct Reynolds numbers (DNS results). (b) Steady-state values of the kinetic energy as a function of the Reynolds number $R$. All DNS results are measured as averages during the statistically steady state. Also shown are model prediction (9) and results from EDQNM closure integration. (c) Same plots as in (b) but in doublelogarithmic representation, plotted as a function of $R-R_{c}$.

certain simulations were observed to suddenly blow up. The reason for this is not completely clear, even though the instability of the linear forcing scheme was mentioned in previous investigations and remedies for this were proposed [6]. We do not implement such remedies, since the linear character of the forcing scheme is a key issue in the present investigation. Whether there is a physical reason behind these instabilities, for instance, the formation of a large-scale two-dimensional structure, can be an interesting issue for further investigation. 
We managed to obtain converged statistics for all Reynolds numbers during the intermediate statistically steady state, except for the interval $1<R<2$. The reason for the instability in this interval will be shown below to be directly related to the triadic nature of the nonlinearity of turbulence, and the discretization of the simulations.

In Fig. 1(b) we show the steady-state values of the kinetic energy as a function of the Reynolds number. A first observation is that the linear forcing scheme leads to large temporal fluctuations around the statistically steady state (as observed and analyzed in Ref. [6] at larger Reynolds numbers). These fluctuations result in substantial error bars. For large values of $R$ the kinetic energy saturates at a value of $K \approx 16$. Furthermore, we see that for low values of $R$ the kinetic energy drops. However, this drop is not monotonous, but a local maximum is observed around $R=5$. This maximum was not anticipated in the prediction following from Model 1 [Eq. (9)], which is also shown in the figure. The model yields by construction the correct critical Reynolds number and asymptotic largeReynolds-number value for $K$. However, for all intermediate values the model does not describe the data so well.

Also shown in the figure are closure results which follow the DNS data quite accurately. Also the EDQNM results display a bump, but somewhat less pronounced. The EDQNM results do not blow up for $1<R<2$, and it is shown in Fig. 1(c) that the closure results approach the critical Reynolds number following a linear relation, proportional to $\left(R-R_{c}\right)$, whereas Model 1 yields a $\left(R-R_{c}\right)^{2}$ power law in this limit.

In Fig. 2 we show the kinetic energy spectra. The EDQNM results (a) and DNS results (b) show a very similar behavior. For the smallest values of the Reynolds number, just beyond $R=1$, the spectrum obtained from the closure integrations consists of a peak, so that the energy spectrum can be written in this limit

$$
E(\kappa) \approx K \delta\left(\kappa-\kappa_{0}\right)
$$

For larger values of $R$, a broad-band spectrum develops. At the largest values considered here $(R=100)$, the beginning of a small inertial range is observed.

In Fig. 3 we show the normalized dissipation rate, $\epsilon L / K^{3 / 2}$, as a function of $R_{K}$. Note that in the present case, since $\epsilon=2 \alpha K$, the normalized dissipation $\epsilon L / K^{3 / 2}=2 \alpha L / K^{1 / 2}$. We observe that the asymptotic behavior is as expected, with a low-Reynolds-number scaling $\sim C_{v} / R_{K}$, with $C_{v}=2$ and a constant value $C_{\epsilon} \approx 0.5$ for large $R_{K}$. It is also observed that the bump noticed in the kinetic energy [Fig. 1(b)] corresponds to an undershoot below the asymptotic value $C_{\epsilon}$. Model 1 is shown to give a too gradual transition between the two asymptotic values, as for the kinetic energy, and does not capture the undershoot.

In the following we will analytically investigate the detailed dynamics of the system near the onset of turbulence. This analysis will allow us to

(i) Explain the physical reason for the absence of steady states in the range $1<R<2$ for the DNS

(ii) Explain the local maximum (or bump) in the kinetic energy

(iii) Understand the asymptotic behavior of $K$ for $R \downarrow R_{c}$ and

(iv) Refine the dissipation model.

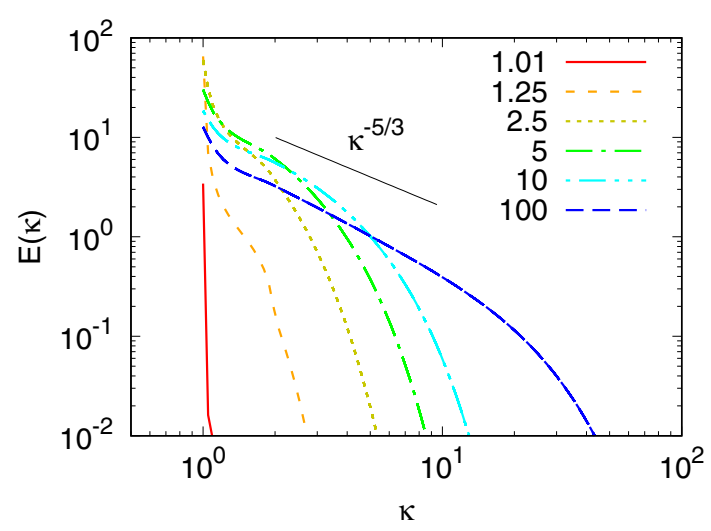

(a)

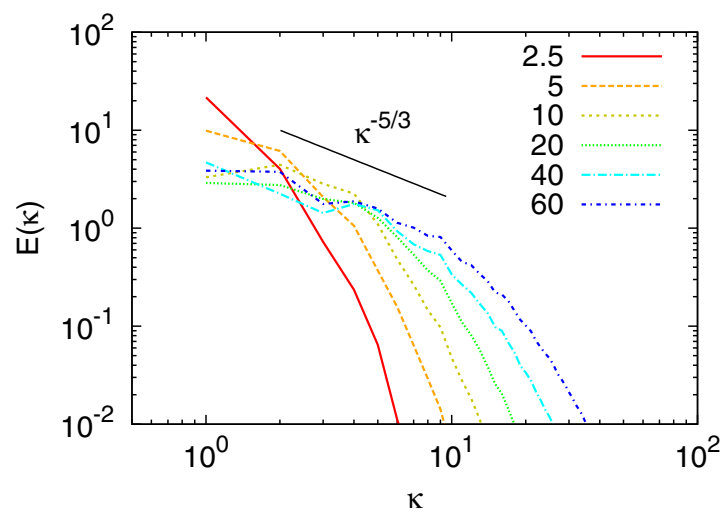

(b)

FIG. 2. Steady-state kinetic energy spectra. (a) EDQNM results for Reynolds numbers $[1.01 \leqslant R \leqslant 100]$. For the lowest Reynolds number the spectrum consists of a peak around $\kappa=\kappa_{0}=1$, while for the largest values of $R$ the beginning of an inertial range starts to emerge. (b) DNS results for Reynolds numbers $[2.5 \leqslant R \leqslant 60]$.

\section{THE DISSIPATION RATE CLOSE TO THE CRITICAL REYNOLDS NUMBER}

The dissipation model [Model 1, Eq. (6)] was shown not to accurately fit the data. Indeed its asymptotic behavior is correct for both low and high Reynolds numbers $R_{K}$, but for

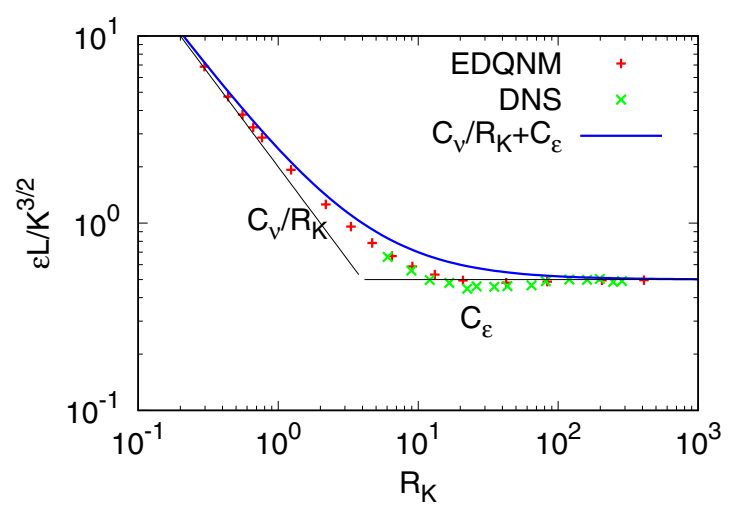

FIG. 3. Normalized dissipation rate as a function of the kineticenergy-based Reynolds number $R_{K}$ (DNS results and EDQNM results). Also plotted is the approximation resulting from Model 1. 
intermediate values it does not very accurately describe the current results for linearly forced turbulence. This results in an incorrect asymptotic behavior of the value of the kinetic energy for low Reynolds numbers.

The model contains two terms: a linear term, containing the constant $C_{\nu}$, which sets the critical Reynolds number, and a nonlinear contribution, containing $C_{\epsilon}$, which allows the saturation of the value of the kinetic energy. In this section we will first focus on the linear contribution to the dissipation for small Reynolds numbers. Then we will consider the nonlinear contribution. It is shown that in order to fit the data more precisely in this limit, both contributions need to be adapted. A refined model is proposed which takes into account these modifications.

\section{A. Low-Reynolds-number behavior of the dissipation-rate equation}

The exact low-Reynolds-number behavior of the dissipation can be derived by considering the spectral dynamics of the dissipation rate equation. This equation is obtained by multiplying the Lin equation (11) by $2 \nu \kappa^{2}$ and integrating,

$$
d_{t} \epsilon=2 \alpha \epsilon+2 \nu \int_{0}^{\infty} \kappa^{2} T(\kappa) d \kappa-4 v^{2} \int_{0}^{\infty} \kappa^{4} E(\kappa) d \kappa
$$

It was observed in Fig. 2, that near the onset the kinetic energy spectrum is close to a $\delta$ pulse at $\kappa=\kappa_{0}$ [see also Eq. (26)]. If all energy is concentrated at one single frequency, the nonlinear transfer is small so that during a steady state the first and the last terms on the right-hand side of Eq. (27) are dominant. Using expression (26), this yields

$$
\epsilon \approx 2 \frac{v^{2}}{\alpha} \kappa_{0}^{4} K=2 R^{-1} \frac{v}{L^{2}} K
$$

This expression suggests that close to the onset, the linear contribution to Model 1 should be modified by a factor $R^{-1}$,

$$
\epsilon=\frac{K^{3 / 2}}{L}\left(C_{\epsilon}+\frac{C_{v}^{\prime}}{R_{K}} R^{-1}\right), \quad(\operatorname{Model} 2 \mathrm{a})
$$

with $C_{v}^{\prime}=2 R_{c}^{2}$. We further note that the linear part of the dissipation is negligible for large $R$ so that we will use this modification for all Reynolds numbers. Model 2a has the same low and high Reynolds number limits as Model 1, but refines this expression close to the onset. Using Model 2a the prediction for the kinetic energy becomes

$$
\frac{K}{(\alpha L)^{2}}=\frac{4}{C_{\epsilon}^{2}}\left[1-\left(\frac{R_{c}}{R}\right)^{2}\right]^{2}
$$

where $R_{c}$ remains thus unity in our setup. The expression is similar in shape to equation (9), but the factor $R_{c} / R$ is now squared. We show the performance of Model 2a in Fig. 4. Clearly the refinement allows a much better agreement with the data for Reynolds numbers $R-R_{c} \gtrsim 1$. However, the bump is absent and the low-Reynolds-number asymptotic scaling is still not reproduced.

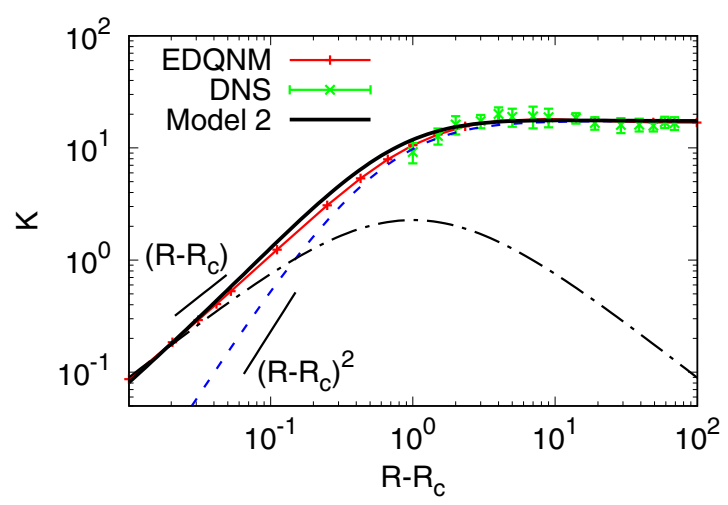

FIG. 4. Comparison of the refined model, Model 2 [resulting from Eq. (45)] with the DNS and EDQNM results. Also shown are the results corresponding to Model 2a [Eq. (29), blue dashed line] and Model 2b [Eq. (43) black dash-dotted line].

\section{B. Triadic interactions near the onset}

We saw in Fig. 1(c) that close to the onset $K \sim\left(R-R_{c}\right)$, which was not reproduced by the simple model (Model 1). The refinement proposed in the last section (Model 2a) yields better agreement with the data, but does not improve the results on this point. In order to change the low kinetic energy behavior of the model, it is thus not enough to modify the linear contribution to the dissipation, but one needs to change the shape of the nonlinear contribution. In order to understand the physics behind the low-Reynolds-number asymptotic behavior of the kinetic energy, we will assess the quasinormal approximation near the onset.

Indeed, for $K \downarrow 0$ the EDQNM approximation changes its nature. In this limit the eddy damping [the $\eta$ contributions in expression (24)] becomes negligible compared to the viscous term. We end up in this limit with a quasinormal approximation, which should rather accurately describe the statistics of the low-Reynolds-number Navier-Stokes system, since the influence of the most drastic assumption, the eddy damping, becomes negligible in this limit.

In the low-Reynolds number limit, where only the mode with largest wavelength (or lowest wave number) is unstable, the spectrum is dominated by a $\delta$ peak (see Fig. 2),

$$
E(\kappa)=E_{0}(\kappa)+\tilde{E}(\kappa),
$$

where $\int_{0}^{\infty} \tilde{E}(\kappa) d \kappa \ll K$, and

$$
E_{0}(\kappa) \approx K \delta\left(\kappa-\kappa_{0}\right)
$$

Clearly, if we are interested in nonlinear effects, it is not enough to consider $E_{0}(\kappa)$ only, as was done in the foregoing, since a single mode cannot lead to nonlinear transfer. We also need to take into account, to leading order, the interaction with $\tilde{E}(\kappa)$.

We decompose the nonlinear transfer into two parts,

$$
T(\kappa)=T^{+}(\kappa)-T^{-}(\kappa)
$$


where

$$
\begin{aligned}
& T^{+}(\kappa)=\iint_{\Delta} f(\kappa, p, q) \frac{\kappa^{2} E(q) E(p)}{q} d p d q, \\
& T^{-}(\kappa)=\iint_{\Delta} f(\kappa, p, q) \frac{p^{2} E(q) E(\kappa)}{q} d p d q,
\end{aligned}
$$

with

$$
f(\kappa, p, q)=\left(x y+z^{3}\right) \Theta(\kappa, p, q) .
$$

The contributions correspond physically to an absorption term $T^{+}(\kappa)$, which represents how mode $\kappa$ receives energy from the other modes, and an emission term $T^{-}(\kappa)$ associated with energy transfer from mode $\kappa$ to the other modes [26]. Typically, since the dominant energetic mode is $E\left(\kappa_{0}\right)$, it is important to know how this mode emits energy to the other modes associated with $\tilde{E}(\kappa)$, which is represented by the term $T^{-}(\kappa)$.

In order to evaluate the low-Reynolds-number behavior of the kinetic energy, we write therefore the dynamics of $E_{0}(\kappa)$,

$$
\partial_{t} E_{0}(\kappa) \approx 2 \alpha E_{0}(\kappa)-2 v \kappa^{2} E_{0}(\kappa)-T^{-}(\kappa) .
$$

Indeed, it is observed that $T^{+}\left(\kappa_{0}\right)$ does not significantly exchange energy with other modes, since the only leading order terms are $\kappa=p=q=\kappa_{0}$. Physically speaking, the energy which $E_{0}\left(\kappa_{0}\right)$ receives from $\tilde{E}(\kappa)$ is negligible compared to the energy $E_{0}\left(\kappa_{0}\right)$ gives to it. The low-Reynolds-number evolution of the kinetic energy is obtained by integrating equation (37), using (32), leading to

$$
d_{t} K=2 \alpha K-2 v \kappa_{0}^{2} K-\epsilon_{0},
$$

where $\epsilon_{0}$ is given by

$$
\epsilon_{0}=\int T^{-}(\kappa) d \kappa
$$

We can simplify further. The dominant contribution to $\epsilon_{0}$ comes from $E_{0}\left(q=\kappa_{0}\right)$ and $E_{0}\left(\kappa=\kappa_{0}\right)$, yielding

$$
\begin{aligned}
\epsilon_{0} & =K^{2} \int_{\kappa_{0}}^{\infty} \iint_{\Delta} f(\kappa, p, q) \frac{p^{2} \delta\left(q-\kappa_{0}\right) \delta\left(\kappa-\kappa_{0}\right)}{q} d p d q d \kappa \\
& =K^{2} \int_{\kappa_{0}}^{2 \kappa_{0}} f\left(\kappa_{0}, p, \kappa_{0}\right) \frac{p^{2}}{\kappa_{0}} d p .
\end{aligned}
$$

and the integration domain, where triangles can be formed with sides $\kappa_{0}, p, \kappa_{0}$ gives possible values of $\kappa_{0}<p \leqslant 2 \kappa_{0}$. The factor $f\left(\kappa_{0}, p, \kappa_{0}\right)$, in the limit $\nu \kappa_{0}^{2} \gg \eta(\kappa)$ for all $\kappa$, writes

$$
f\left(\kappa_{0}, p, \kappa_{0}\right)=\frac{\left(x y+z^{3}\right)}{v\left(2 \kappa_{0}^{2}+p^{2}\right)} .
$$

Introducing $\gamma=p / \kappa_{0}$ and using the cosine rule, we find the values $x=z=\gamma / 2, y=1-\gamma^{2} / 2$, so that

$$
\begin{aligned}
\epsilon_{0} & =\frac{K^{2}}{8 v} \int_{1}^{2} \frac{\gamma^{3}\left(4-\gamma^{2}\right)}{\left(\gamma^{2}+2\right)} d \gamma \\
& =C_{0} \frac{K^{2}}{v}
\end{aligned}
$$

We compute the integral and find $C_{0}=21 / 32-$ $(3 / 4) \ln (2) \approx 0.136$. This corresponds to an expression for the dissipation rate,

$$
\epsilon=\frac{K^{3 / 2}}{L}\left(C_{v} R_{K}^{-1}+C_{0} R_{K}\right), \quad(\text { Model 2b) }
$$

giving steady-state solutions $K=0$ and

$$
\frac{K}{\alpha^{2} L^{2}}=\frac{2}{C_{0} R}\left(1-\frac{R_{c}}{R}\right)
$$

This expression, for small values of $K$, behaves as $K \sim(R-$ $R_{c}$ ), as observed in the closure integration. In Fig. 4 we show that the small-Reynolds-number limit of this model is indeed correct. We have used the value $C_{0}=0.22$, which is somewhat larger than the analytical value, but which fits the data better. At large Reynolds numbers, the model obviously fails, since the influence of nonlinear scrambling (or eddy damping) $\eta(\kappa)$ cannot be neglected anymore compared to the viscous damping $\nu \kappa^{2}$.

We close this section by elucidating why the DNS cannot reproduce steady states for $1<R<2$. Indeed, it is shown in expression (40) that the dominant mode $E\left(\kappa_{0}\right)$ can only interact with modes where $\kappa \leqslant 2 \kappa_{0}$. In the DNS the discretization of the modes is such that no modes are present in this interval. The dominant mode can therefore not exchange energy and, in the absence of nonlinear saturation, the simulation is bound to blow up. For $R=2$ the secondmost unstable mode $E\left(2 \kappa_{0}\right)$ is excited, such that from this point on, nonlinear interactions are allowed by the system. In the closure, where a continuous spectrum of modes is considered, this effect is not present and the kinetic energy can continuously approach zero for $R \downarrow R_{c}$. We note here that this discrete nature of the triadic interactions is also leading to the recently discovered staircase scaling of short-time energy transfer [27].

\section{Refined model}

In order to combine the two dissipation rate expressions (Model 2a and Model 2b) we write the expression

$$
\epsilon=C_{\nu} \frac{v}{L^{2}} R^{-1} K+C_{\epsilon} \frac{K^{3 / 2}}{L} \frac{1}{C_{1} R_{K}^{-1}+1}, \quad \text { (Model 2) }
$$

or equivalently, for the normalized dissipation rate

$$
\frac{\epsilon L}{K^{3 / 2}}=C_{v}\left(R_{K} R\right)^{-1}+\frac{C_{\epsilon}}{C_{1} R_{K}^{-1}+1},
$$

with $C_{1}$ a constant. The solution for the kinetic energy given by this model is

$$
\frac{K}{(\alpha L)^{2}}=\left\{\Xi(R)+\left[\Xi(R)^{2}+\frac{2 C_{1}}{R} \Xi(R)\right]^{1 / 2}\right\}^{2}
$$

with

$$
\Xi(R)=\frac{1}{C_{\epsilon}}\left(1-R_{c} / R^{2}\right)
$$

Using the values $C_{1}=0.7$ and $C_{\epsilon}=0.5$ (and $C_{v}=2$ ), it is observed in Fig. 4 that this model reproduces quite accurately the kinetic energy for all Reynolds numbers. This model contains the bump in the kinetic energy which is explained as the intersection of the two asymptotic regimes (30) and (44). 
In particular, near the onset the kinetic energy is given by $K \sim\left(R-R_{c}\right)$.

The bump is less pronounced than in the DNS or closure for the parameters $C_{\epsilon}, C_{1}$ which give the best fit of the asymptotic regimes. Further refinement of the model to improve the agreement does not seem desirable, since it would probably require nonlocal-triadic effects and the model would not be much simpler than the EDQNM closure equations themselves.

\section{CONCLUSION}

We have investigated linearly forced isotropic turbulence. We show that a critical Reynolds number exists, $R_{c}=L^{2} \kappa_{0}^{2}$, with $\kappa_{0}$ the most unstable mode and $L$ a typical length scale. At this value of the Reynolds number the solution bifurcates from zero to nonzero kinetic energy. The transition is of supercritical nature, where here the base state is a trivial zero-velocity state. This can be contrasted with the results of the investigation [1] where the use of localized spectral forcing leads to a typical subcritical transition. The transition to turbulence, or the laminarization, in isotropic turbulence is thus not universal, but depends on the type of forcing.

A detailed analysis of the triad interactions shows that a single-wave-number energy distribution will allow interactions only with modes of maximum twice the excited wave number. In pseudospectral DNS, where the discretization is imposed by the base functions this results in an impossibility to attain a nontrivial steady state for Reynolds numbers in the range $1<R<2$ for linearly forced turbulence.

For the present case of linear forcing, a new dissipation model is derived and this model, Eq. (45), is shown to give an improved agreement for the dependence of the dissipation and the kinetic energy on the Reynolds number. It is perhaps not the exact form of this model which is the important result here, but the insight how the kinetic energy behaves close to the onset, i.e., $K \sim R-R_{c}$.

It should be mentioned that just beyond the critical Reynolds number, where the energy spectrum is close to a $\delta$ peak, the flow should perhaps not be called turbulent. In our system, it seems that the development to a broad-band spectrum takes place in a continuous way. It would be interesting to assess in detail the physical-space structure of the flow beyond the onset. Indeed, in the case of transitional shear flows near the onset, such as plane Couette flow, the physical space structure shows an intricate pattern of stripy turbulent regions embedded in a laminar flow (see, for instance, Ref. [28] or the recent review [29]). An investigation of this for the present case is left as a perspective for further research.

The present findings are interesting in the light of recent advances on the understanding of transition in shear flow. Indeed the present work is motivated by the recent insight that the transition in shear flows bounded from two sides, such as plane-Poiseuille flow and plane-Couette flow, might be continuous, and that the discontinuous behavior around the critical Reynolds number is probably due to finite-size effects [29-32]. The obtained results in the present investigation, which are relatively easy to understand, can then serve as a test case of, perhaps, the simplest possible turbulent system exhibiting a continuous transition, against which more complicated flows such as the above mentioned shear flows can be assessed. For instance, it is observed that in channel flow in the transitional regime, where laminar and turbulent flow coexist, the turbulent volume-fraction can approximately be described by a two-dimensional directed percolation model $[29,31]$. However, very close to the onset this description breaks down [33]. Possibly, the present approach might be transposable to channel flow, so that in such flow, at very low Reynolds numbers $R_{K}$, the kinetic energy could also be damped by a nonlinear saturation piloted by a viscous timescale $L^{2} / v$, as represented by Model $2 \mathrm{~b}$. Such an investigation is currently in progress.

\section{ACKNOWLEDGMENTS}

The authors gratefully acknowledge the insightful comments of Yohann Duguet. We further thank the referees for interesting suggestions. This work was performed using HPC resources from the FLMSN (Fédération Lyonnaise de Modélisation et Sciences Numériques), partner of EQUIPEX EQUIP@MESO.
[1] M. F. Linkmann and A. Morozov, Phys. Rev. Lett. 115, 134502 (2015).

[2] K. R. Sreenivasan, Acta Mech. 44, 1 (1982).

[3] T. Lundgren, Annual Research Briefs-2003 (Center for Turbulence Research, Stanford University, 2003), p. 461.

[4] D. D. Joseph, Stability of Fluid Motions I (Springer, New York, 1976).

[5] C. Rosales and C. Meneveau, Phys. Fluids 17, 095106 (2005).

[6] P. L. Carroll and G. Blanquart, Phys. Fluids 25, 105114 (2013).

[7] J. Kim and J. Lim, Phys. Fluids 12, 1885 (2000).

[8] J. C. Del Álamo and J. Jiménez, J. Fluid Mech. 559, 205 (2006).

[9] A. Lozano-Durán, N. C. Constantinou, M.-A. Nikolaidis, and M. Karp, arXiv:2005.05303 (2020).
[10] W. D. McComb, A. Berera, S. R. Yoffe, and M. F. Linkmann, Phys. Rev. E 91, 043013 (2015).

[11] G. I. Taylor, Proc. Roy. Soc. London. Ser. A, Math. Phys. Sci. 151, 421 (1935).

[12] L. Onsager, Il Nuovo Cimento 6, 279 (1949).

[13] Y. Kaneda, T. Ishihara, M. Yokokawa, K. Itakura, and A. Uno, Phys. Fluids 15, L21 (2003).

[14] W. J. T. Bos, L. Shao, and J. P. Bertoglio, Phys. Fluids 19, 045101 (2007).

[15] M. F. Linkmann, J. Fluid Mech. 856, 79 (2018).

[16] P. C. Valente and J. C. Vassilicos, Phys. Rev. Lett. 108, 214503 (2012).

[17] W. J. T. Bos and R. Rubinstein, Phys. Rev. Fluids 2, 022601(R) (2017).

[18] C. R. Doering and C. Foias, J. Fluid Mech. 467, 289 (2002). 
[19] C. R. Doering, B. Eckhardt, and J. Schumacher, J. Fluid Mech. 494, 275 (2003).

[20] P. Garaud and G. I. Ogilvie, J. Fluid Mech. 530, 145 (2005).

[21] M. F. Linkmann, A. Berera, W. D. McComb, and M. E. McKay, Phys. Rev. Lett. 114, 235001 (2015).

[22] J. Mathieu and J. F. Scott, An Introduction to Turbulent Flow (Cambridge University Press, Cambridge, 2000).

[23] S. A. Orszag, J. Fluid Mech. 41, 363 (1970).

[24] W. J. T. Bos and R. Zamansky, Phys. Rev. Lett. 122, 124504 (2019).
[25] H. Touil, J. P. Bertoglio, and L. Shao, J. Turbul. 3, N49 (2002).

[26] R. H. Kraichnan, J. Fluid Mech. 5, 497 (1959).

[27] L. Fang, T. Wu, and W. J. T. Bos, J. Turbul. 21, 234 (2020).

[28] Y. Duguet, P. Schlatter, and D. S. Henningson, J. Fluid Mech. 650, 119 (2010).

[29] L. S. Tuckerman, M. Chantry, and D. Barkley, Annu. Rev. Fluid Mech. 52, 343 (2019).

[30] Y. Pomeau, Physica D 23, 3 (1986).

[31] M. Sano and K. Tamai, Nat. Phys. 12, 249 (2016).

[32] B. Eckhardt, Physica A 504, 121 (2018).

[33] M. Shimizu and P. Manneville, Phys. Rev. Fluids 4, 113903 (2019). 